\title{
OAM Beam Generation using All-fiber Fused Couplers
}

\author{
S. Pidishety ${ }^{1,2 *}$, M.I.M. Abdul Khudus ${ }^{2,4}$, P. Gregg ${ }^{3}$, S. Ramachandran ${ }^{3}$, B. Srinivasan ${ }^{1}$, and G. Brambilla ${ }^{2}$ \\ 1. Department of Electrical Engineering, Indian Institute of Technology Madras, Chennai-600036, India \\ 2. Optoelectronics Research Centre, University of Southampton, Southampton, SO171BJ, United Kingdom \\ 3. Electrical and Computer Engineering Department, Boston University, 8St Mary's St, Boston, MA, USA \\ 4. Photonics Research Centre, Department of Physics, Faculty of Science, University of Malaya, 50603-Kuala Lumpur, Malaysia \\ *Email: pd.shankara@gmail.com
}

\begin{abstract}
We demonstrate the orbital angular momentum (OAM) beam generation using an allfiber fused coupler based on single mode fiber (SMF) and air-core fiber. The fabricated device is directly SMF compatible with $\sim 80 \%$ power coupling efficiency.

OCIS codes: (060.2310) Fiber optics; (230.1150) Optical devices; (050.4865) Optical vortices
\end{abstract}

\section{Introduction}

Optical beams with orbital angular momentum (OAM), characterized by a helical phase front, $\exp (i l \phi)(l \& \phi$ being topological charge and azimuthal angle, respectively), have gained much interest in recent years due to the potential for using OAM states as orthogonal signal channels for scaling the bandwidth of next-generation optical communication networks [1-3]. OAM generation has been widely studied in free space [1, 4, 5 and references therein], silicon ships $[6,7]$, and to a limited extent, in fiber $[8,9]$. While a few of these devices offer scalability, i.e. ability to multiplex many OAM modes with low loss, none of them are directly fiber compatible. Hence, alternative all-fiber based techniques which offer the potential for direct integration with existing telecom/datacom infrastructures, and yet are mode-scalable, are highly desirable.

In this paper, we demonstrate generation of OAM beam through the well-known fused fiber coupler fabricated using single mode fiber (SMF) and OAM air-core fiber [10, 11], by using the mechanism of exciting specific higher order modes (HOM) [12, 13]. As fused fiber couplers as well as photonic lanterns [14] have been the widely utilized in Mode Division Multiplexing (MDM) transmission, the demonstrated all-fiber based fused coupler provides the first realistic pathway to multiplexing many OAM modes in fibers with low loss.

\section{Fused fiber couplers and excitation of OAM beam}

In general, modes in the air-core fibers propagate with different effective indices $\left(\mathrm{n}_{\mathrm{eff}}\right)$ than $\mathrm{LP}_{01}$ in $\mathrm{SMF}$, due to the different fiber refractive index profiles. The $\mathrm{n}_{\mathrm{eff}}$ or propagation constants of the selected modes must be matched in order to achieve coupling from $\mathrm{LP}_{01}$ in SMF to any HOM in the air-core fiber [13]. As HOMs are associated with lower $\mathrm{n}_{\mathrm{eff}}$, the diameter of the SMF is reduced, by pre-tapering, to phase match $\mathrm{LP}_{01}$ with that of a selected HOM in the air-core fiber.
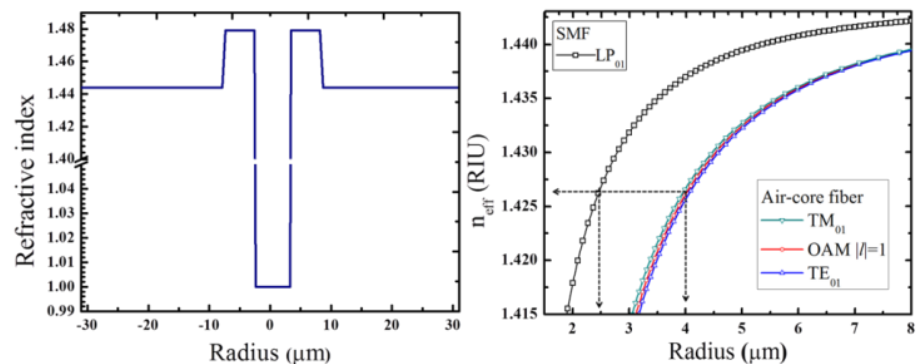

Figure 1: Simulation results: (a): Index profile of OAM air-core fiber, (b): Effective index $\left(\mathrm{n}_{\text {eff }}\right)$ of different modes in $\left.\mathrm{SMF}_{(\mathrm{LP}} \mathrm{LP}_{01}\right)$ and air-core fiber $\left(\mathrm{TM}_{01}, \mathrm{OAM}|l|=1, \mathrm{TE}_{01}\right)$ fiber as a function of radius of fiber; phase matching points are indicated.

The air-core fiber, shown in Fig. 1. (a), ensures stable propagation of excited OAM modes [10, 11] by lifting the modal degeneracy and suppressing the intermodal cross talk. The phase matching condition is studied using COMSOL Multiphysics ${ }^{\circledR}$ eigen mode solver to estimate the diameter ratio between SMF and OAM fiber. Fig 1. (b), shows $\mathrm{n}_{\mathrm{eff}}$ for the $\mathrm{LP}_{01}$ mode in $\mathrm{SMF}$ and the OAM $|l|=1$ mode in the air-core fiber are mapped as a function of fiber radius in order to calculate fiber diameter ratio to achieve phase matching [8]. When phase matching is achieved, the superposition of excited OAM $|l|=1$ modes is tuned by controlling input polarization, with each circular polarization exciting one specific OAM mode in its 2-mode degenerate subspace.

\section{Experimental details, results and discussion}

Conventional telecom-grade SMF is pre-tapered to $\sim 75.8 \mu \mathrm{m}$ (with less than $0.1 \mathrm{~dB}$ loss) according to the diameter ratio calculated from simulation studies (Fig. 1. (b)). The pre-tapered SMF is longitudinally aligned with the un- 
tapered air-core fiber without any twist, and fixed with a UV-curable adhesive glue. The coupler is fabricated using the modified flame brushing technique by fusing both the fibers at $\sim 1400{ }^{\circ} \mathrm{C}$ using a ceramic micro heater (NTT-AT, Japan) and by pulling them using a commercial fiber tapering rig. After a certain length of pulling, the fibers weakly fuse and the $\mathrm{LP}_{01}$ mode in the SMF couples into the OAM mode of the air-core fiber to which it is phase matched. The tapering is stopped when power measured out of the air-core fiber is maximized.

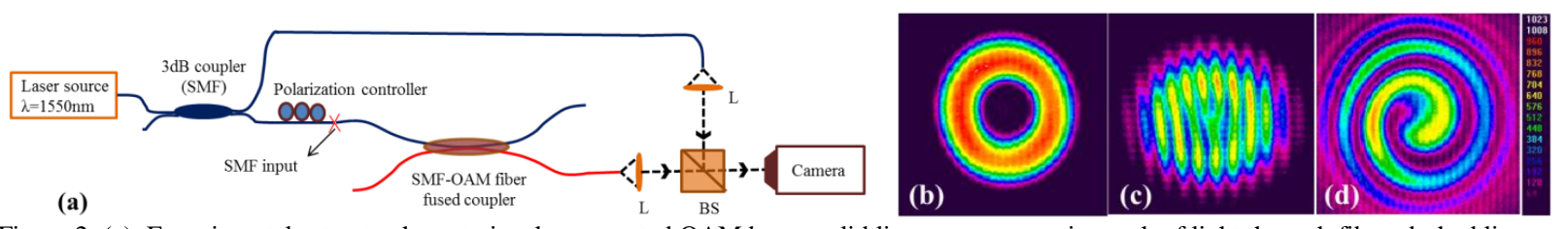

Figure 2: (a): Experimental setup to characterize the generated OAM beam-solid lines are propagation path of light through fiber, dashed lines are that of light through free space, L-collimating lens, BS-free space beam splitter. (b): Far field image of the generated OAM beam. (c) \& (d): Fork and spiral interference patterns of OAM beam and Gaussian reference, respectively.

Fig. 2. (a) depicts the schematic for device characterization: light from a 1550-nm laser source (linewidth $\sim 0.4 \mathrm{~nm}$ ) is split into two arms using a $3 \mathrm{~dB}$ coupler. One arm is passed through a polarization controller and spliced to the fabricated OAM coupler, and the other arm is used to construct and SMF-based reference arm in the interference setup. The output beams from the reference SMF and air-core fiber (cleaved within $\sim 30 \mathrm{~cm}$ after the coupling region in the device) are collimated and then interfered using a free space beam splitter. The field patterns are imaged using a CCD camera (MicronViewer-7290A). Fig. 2. (b) shows a clear doughnut pattern out of the aircore fiber output when the reference arm is blocked. The absence of $\mathrm{LP}_{11}$ mode like patterns and a relatively uniform azimuthal intensity distribution qualitatively suggests that phase matched coupling to the OAM modes was achieved without substantial coupling to the neighboring $\mathrm{HE}_{11}, \mathrm{TE}_{01}$ or $\mathrm{TM}_{01}$ modes (see Fig. 1. (b) for mode designations). A polarization measurement performed using a polarizer and quarter wave plate combination reveals that this beam is uniformly circularly polarized, further suggesting that these beams are OAM eigenmodes of the air-core fiber. With the reference arm un-blocked but incident on the camera at a slight angle with respect to the beam from the air-core fiber, the input polarization is adjusted until the characteristic fork interference pattern is observed (Fig. 2. (c)). With the beam from the reference arm co-aligned with that from the air-core fiber, we observe the characteristic spiral interference between an OAM and an expanded Gaussian beam (Fig. 2. (d)). These observations are consistent with the fact that the beam exiting the air-core fiber is substantially a pure OAM mode. The coupling efficiency of the device is measured to be $\sim 80 \%$, and further studies on quantitatively determining the purity of output OAM beams and enhancing the efficiency of the device are under progress.

In summary, we demonstrate the first, to the best of our knowledge, fused fiber coupler that enables generation of OAM beams in fibers that stably propagate them, using an SMF input. Since this is a side coupling technique, it could potentially be repeated along the length of the OAM fiber to simultaneously generate multiple OAM orders, thereby yielding a fiber-compatible, low loss, scalable (de)multiplexer of OAM beams, which are of immense interest to OAM-based capacity enhancement schemes.

S. Pidishety would like to acknowledge the financial support for his visit to ORC through the IRSES grant (PIRSES-GA-2012-318941) and AFOSR, and Vijay Kumar, Physical Research Laboratory, Ahmedabad, Gujarat, India, for fruitful discussion.

\section{References}

[1] A.M. Yao, M.J. Padgett, "Orbital angular momentum: origins, behavior and applications," Adv. Opt. Photonics. 3 161-204 (2011).

[2] J. Wang et al, "Terabit free-space data transmission employing orbital angular momentum multiplexing," Nat. Photonics. 6 488-496 (2012).

[3] N. Bozinovic et al, "Terabit-Scale Orbital Angular Momentum Mode Division Multiplexing in Fibers," Science, 340, $1545-1548$ (2013).

[4] L. Marrucci, et al, "Optical Spin-to-Orbital Angular Momentum Conversion in Inhomogeneous Anisotropic Media," Phys. Rev. Lett. 96, 163905-4 (2006).

[5] G. C. G. Berkhout et al., "Efficient Sorting of Orbital Angular Momentum States of Light," Phys. Rev. Lett. 105, 15360-4 (2010).

[6] X. Cai et al, "Integrated Compact Optical Vortex Beam Emitters," Science. 338 363-366 (2012).

[7] J. Sun $e t$ al, "Generating and identifying optical orbital angular momentum with silicon photonic circuits", Opt. Lett. 39 5977-5980 (2014).

[8] S. Ramachandran et al, "Generation and propagation of radially polarized beams in optical fibers", Opt. Lett. 34 2525-2527 (2009).

[9] T. Grosjean, D, et al, "An all-fiber device for generating radially and other polarized light beams," Opt. Commun. 203, 1-5 (2002).

[10]C. Brunet et al., "Design, fabrication and validation of an OAM fiber supporting 36 states," Opt. Express 22, 26117-26127 (2014).

[11] P. Gregg, et al, "Conservation of orbital angular momentum in air-core optical fibers". Optica. 2, 267-270 (2015

[12] R. Ismaeel et al, "All-fiber fused directional coupler for highly efficient spatial mode conversion," Opt. Express, 22, 11610-11619 (2014).

[13] G. Pelegrina-B et al., "Analysis of the Coupling Mechanism in Asymmetric Fused Fiber Couplers," J. Light. Technol. 32, 2382-2391 (2014).

[14] T. A. Birks et al., “The photonic lantern,” Adv. Opt. Photonics 7, 107-167 (2015). 